\title{
Stable Equivalence of the Weak Closures of Free Groups Convolution Algebras
}

\section{Florin Rădulescu}

Department of Mathematics, U.C.L.A., Los Angeles, CA 90024, USA and I.H.E.S., 35 Route de Chartres, 91440 Bures-sur-Yvette, France. Present address: Department of Mathematics, University of California, Berkeley, CA 94720, USA. Permanent address: Institute of Mathematics of the Romanian Academy, Str. Academiei 14, Bucharest, Romania

Received January 20, 1992; in revised form October 22, 1992

\begin{abstract}
We prove in this paper that the von Neumann algebras associated to the free non-commutative groups are stably isomorphic, i.e. that they are isomorphic when tensorized by the algebra of all linear bounded operators on a separable, infinite dimensional Hilbert space. This gives positive evidence for an old question, due to R.V. Kadison (see also S. Sakai's book on W*-algebras), whether the von Neumann algebras associated to free groups are isomorphic or not.
\end{abstract}

In this paper we show that the algebras $\mathscr{L}\left(F_{N}\right)=\overline{\mathbb{C}\left(F_{N}\right)^{w}}$, the weak closures of the group algebras associated to free (nonabelian) groups $F_{N}, N \geqq 2, N$ finite, are all stably isomorphic, i.e. that the isomorphism class of $\mathscr{L}\left(F_{N}\right) \otimes B(H)$ doesn't depend on $N \in \mathbb{N}, N \geqq 2$. (Here $B(H)$ is the algebra of bounded operators on an infinite dimensional, separable, Hilbert space $H$ ).

This may serve as evidence for an old problem of R.V. Kadison in the early '60's, on the isomorphism of the algebras $\mathscr{L}\left(F_{N}\right), N \geqq 2$ ([2], see also [9] problem 4.4.44 and [3]).

The first remarkable breakthrough to this end, was the theorem of D. Voiculescu stating that

$$
M_{k}(\mathbb{C}) \otimes \mathscr{L}\left(F_{(N-1) k^{2}+1}\right)
$$

is isomorphic to $\mathscr{L}\left(F_{N}\right), k, N \in \mathbb{N}, N \geqq 2$. (This implies in particular that $\mathscr{L}\left(F_{N}\right)$ and $\mathscr{L}\left(F_{(N-1) k^{2}+1}\right)$ are stably isomorphic for each $k, N \in \mathbb{N}, N \geqq 2$, but it doesn't imply, for example that $\mathscr{L}\left(F_{2}\right)$ and $\mathscr{L}\left(F_{3}\right)$ are stably isomorphic).

Our main tools will be the matrix representation for free families obtained by D. Voiculescu in the setting of noncommutative probability theory $([6,7])$, and the iterative technique of finding generators for reduced free algebras that we used in [4].

In terms of isomorphism classes of reduced algebras, the result of D. Voiculescu was stated as

$$
\mathscr{L}\left(F_{N}\right)_{\frac{1}{k}} \cong \mathscr{L}\left(F_{(N-1) k^{2}+1}\right) ; \quad k, N \in \mathbb{N}, N \geqq 2 .
$$


Here $\mathscr{L}\left(F_{N}\right)_{t}$ is the isomorphism class of the algebra $e \mathscr{L}\left(F_{N}\right) e$, where $e$ is any selfadjoint idempotent in $\mathscr{L}\left(F_{N}\right)=\overline{\mathbb{C}\left(F_{N}\right)^{w}}$, of trace $\tau(e)=t(\tau$ is the weakly continuous linear functional on $\mathscr{L}\left(F_{N}\right)$ defined by $\tau\left(\lambda_{g}\right)=0$ if $g \neq e, \tau\left(\lambda_{e}\right)=1$, where $e$ is the identity, while $\lambda_{g}, g \in F_{N}$ is the left convolution operator on $\mathbb{C}\left(F_{N}\right)$ ).

The proof of our result will come (by usual techniques, see e.g. $[1,5]$ ) from the following isomorphism result (which extends Voiculescu's isomorphism theorem):

$$
\mathscr{L}\left(F_{N}\right)_{\frac{1}{\sqrt{k}}} \cong \mathscr{L}\left(F_{(N-1) k^{2}+1}\right), \quad k, N \in \mathbb{N}, N \geqq 2,
$$

which in particular (taking $N=2$ ), implies that $\mathscr{L}\left(F_{2}\right)$ and $\mathscr{L}\left(F_{k+1}\right)$ are stably isomorphic, $k \in \mathbb{N}$, i.e.

$$
\mathscr{L}\left(F_{2}\right) \bar{\otimes} B(H) \cong \mathscr{L}\left(F_{k+1}\right) \bar{\otimes} B(H) .
$$

In particular our result shows that the following invariant (the fundamental group $([3]) \mathscr{F}\left(\mathscr{L}\left(F_{N}\right)\right)=\left\{t>0 \mid \mathscr{L}\left(F_{N}\right)_{t} \cong \mathscr{L}\left(F_{N}\right)\right\}$ cannot distinguish between the algebras $\mathscr{L}\left(F_{N}\right), N \geqq 2, N$ a positive integer.

To prove the isomorphism relation, we will introduce an extrapolation, with algebras, " $\mathscr{L}\left(F_{r}\right)$ " $r \in \mathbb{Q}, r \geqq 2$ for the series of the algebras $\mathscr{L}\left(F_{N}\right)_{N \in \mathbb{N}, N \geqq 2}$ (such algebras were also considered by $\mathrm{K}$. Dykema (personal communication to the author)). This will be done by the matrix representations for these algebras given by the theorem of D. Voiculescu. The proof will then consist of taking $r$ to the limit, which will be done by the techniques in [4].

In a forthcoming paper ([9]) we will give a meaning to " $\mathscr{L}\left(F_{r}\right)$ " for real $r \geqq 2$ and prove that the formula " $\mathscr{L}\left(F_{r}\right)_{t} \cong \mathscr{L}\left(F_{(r-1) t^{-2}+1}\right)$ " and $\mathscr{L}\left(F_{r}\right) * \mathscr{L}\left(F_{r^{\prime}}\right) \cong$ $\mathscr{L}\left(F_{\left(r+r^{\prime}\right)}\right)$ " are also valid for positive real $t$ and $r$.

I am greatly indebted to G. Skandalis for suggesting this problem. I am also indebted to S. Popa and M. Takesaki for useful comments. I am also indebted to the referee, who pointed out to me a number of minor errors.

This paper has been circulated as an I.H.E.S. preprint, December '91.

\section{Definitions and Outline of the Proof}

Let $H$ be a Hilbert space, $B(H)$ the space of all bounded linear operators acting on $H$. A weakly closed subalgebra $M$ of $B(H)$ is called a von Neumann algebra (or when no reference to $H$ is made, a $W^{*}$-algebra). If $S$ is a subset of $M$, then the von Neumann subalgebra generated in $M$ by $S$ will be denoted by $S^{\prime \prime}$. A von Neumann algebra with no minimal idempotents will be called diffuse.

$M$ is a type $\mathrm{II}_{1}$ factor if it has no center, it is diffuse and there exists a weakly continuous faithful functional $\tau$ on $M$ such that $\tau(x y)=\tau(y x), x, y \in M$. In general we will assume that $\tau$ is normalized, i.e. that $\tau(1)=1$. In this case the strong operator topology on $M$, will be given by the norm $\|\cdot\|_{\tau}$, defined by $\|x\|_{\tau}^{2}=$ $\tau\left(x^{*} x\right)$. This last is a norm since $\tau$ is faithful (i.e. $\tau\left(x^{*} x\right)=0 \Rightarrow x=0$ ).

For $t>0$, the isomorphism class of the von Neumann algebra $e M e$, where $e$ is a selfadjoint idempotent in $M$ with $\tau(e)=t$, depends only on $t$ and is denoted by $M_{t}$. When $t>1$, we replace in this construction $M$ by $M \otimes M_{n}(\mathbb{C})$, where $n$ is a natural number, strictly greater than the integer part of $t$.

A matrix unit $\left(\omega_{i j}\right)_{i, j=1}^{r}$ in $M$ is a family such that $\omega_{i j} \omega_{s k}=\delta_{j s} \omega_{i k}$ (where $\delta_{i s}$ stands for the Kronecker symbol), $i, j, s, k \in\{1,2, \ldots, r\}$. Usually $M_{n}(\mathbb{C}), n \in \mathbb{N}$ 
comes with a canonical normalized trace $\tau_{n}$ and a canonical matrix unit. (We refer to the books $[1,5]$ for an exposition of all the facts recalled before.)

We also recall now some definitions and results from [7]. Let $(M, \varphi)$ be a type $\mathrm{II}_{1}$ factor with trace $\varphi$.

A family of unital subalgebras

$$
\left(A_{i}\right)_{i \in I} \subseteq M
$$

is called a free family if

$$
\varphi\left(a_{1} a_{2} \ldots a_{n}\right)=0
$$

whenever $\varphi\left(a_{j}\right)=0, a_{j} \in A_{i_{j}}, j=1,2, \ldots, n$, with $i_{1} \neq i_{2}, \ldots, i_{n-1} \neq i_{n}$. A family $\left(f_{i}\right)_{i \in I}$ of elements in $M$ will be free if the algebras $\left\{f_{i}, 1\right\}_{i \in I}^{\prime \prime} \subseteq M$ are a free family.

The family $\left(f_{i}\right)_{i \in I}$ is semicircular if it is free and if in addition the trace of the spectral distribution of each element $f_{i}$ is given by the semicircular law:

$$
\varphi\left(f_{i}^{k}\right)=\frac{2}{\pi} \int_{-1}^{1} t^{k}\left(1-t^{2}\right)^{1 / 2} d t, \quad k \in \mathbb{N}, i \in I
$$

A family $\left\{g_{j}\right\}_{j \in J}$ in $M$ will be circular if the family $\left\{x_{j}, y_{j}\right\}_{j \in J}$ is semicircular, where $x_{j}=\frac{g_{j}+g_{j}^{*}}{\sqrt{2}}, y_{j}=\frac{g_{j}+g_{j}^{*}}{\sqrt{2 i}}$.

Finally we recall the following matrix representation (Proposition 2.8 in [7]) of a semicircular family, given by D. Voiculescu.

Let $n \in \mathbb{N}$ be an integer, $S$ a nonempty set and let

$$
\omega_{1}=\{f(i, s) \mid i=1,2, \ldots, n, s \in S\}
$$

be a free semicircular family in $M$, let

$$
\omega_{2}=\{g(i, j, s) \mid 1 \leqq i \leqq j \leqq n, s \in S\}
$$

be a circular family in $M$ and let $a$ be a semicircular selfadjoint element of $M$. Moreover assume that $\omega_{1} \cup \omega_{2} \cup\{a\}$ is a free family.

Consider the following elements in the von Neumann algebra $M \otimes M_{n}(\mathbb{C})$ endowed with the canonical trace $\varphi \otimes \tau_{n}$, where $M_{n}(\mathbb{C})$ has the matrix unit $\left(e_{i j}\right)_{i, j=1}^{n}$,

$$
X^{s}=\frac{1}{\sqrt{n}}\left(\sum_{i=1}^{n} f(i, s) \otimes e_{i i}+\sum_{1 \leqq i<j \leqq n}\left(g(i, j, s) \otimes e_{i j}+g(i, j, s)^{*} \otimes e_{j i}\right)\right)
$$

for $s \in S$. Moreover let $X^{\sigma}$ be any semicircular element in $M \otimes M_{n}(\mathbb{C})$ generating the same von Neumann algebra as $\left\{a \otimes e_{i i} \mid i=1,2, \ldots, n\right\}$.

The Theorem of D. Voiculescu asserts that the family $\left(X^{s}\right)_{s \in S} \cup\left(X^{\sigma}\right)$ $\subseteq M \otimes M_{n}(\mathbb{C})$ is also a free semicircular family. This was the essential step in its proof that

$$
\mathscr{L}\left(F_{N}\right)_{\frac{1}{k}} \simeq \mathscr{L}\left(F_{(N-1) k^{2}+1}\right)
$$

and this will also be the basic fact in our proofs.

Recall that $\mathscr{L}(G)$ is the weak closure of the left convolution algebra of $G$, and that if $G$ is $F_{N}, N \geqq 2$ (in fact if $G$ has infinitely many conjugacy classes) then $\mathscr{L}(G)$ is a type $\mathrm{II}_{1}$ factor [5].

We now introduce some new definitions on free algebras that we will be using in the proof of our results. 
The matrix representation ([7]) of a free semicircular family, makes it natural to introduce an interpolation with rational numbers of the family $\mathscr{L}\left(F_{N}\right), N \geqq 2, N \in \mathbb{N}$, by defining " $\mathscr{L}\left(F_{q}\right)$ " for rational $q \geqq 2$, (although $F_{q}$ has no meaning).

The construction of such an algebra runs as follows: let $\left(X^{0}, X^{1}, \ldots, X^{N}\right)$ be a free semicircular family in $M \otimes M_{n}(\mathbb{C})$, where we use the matrix picture described before with $S=\{1, \ldots, N\}$ and $\sigma=0$. Assume that $q=N+\frac{k}{n^{2}}$, where $k$ is an integer in the set $\left\{0,1, \ldots, n^{2}-1\right\}$ and $N \geqq 2$.

We remove from $X^{N}, k^{\prime}$ diagonal entries of the form $f(i, N) \otimes e_{i i}$, and $k^{\prime \prime}$ upper diagonal entries of the form $f(i, j, N) \otimes e_{i j}$ together with their adjoints. In this way, by removing $k^{\prime}+2 k^{\prime \prime}=n^{2}-k$ entries from $X^{N}$ we obtain a new selfadjoint element in $X^{\prime N}$ in $M \otimes M_{n}(\mathbb{C})$.

We will take as a definition for the algebra $\mathscr{L}\left(F_{q}\right)$, the isomorphism class of the von Neumann algebra $\mathscr{A}$ generated in $M_{n}(\mathbb{C}) \otimes M$, by $X^{0}, X^{1}, \ldots, X^{N-1}, X^{\prime N}$.

The arguments in the proof of Theorem 3.3 in [7], obviously extend to give that

$$
\mathscr{A}_{\frac{1}{n}} \cong \mathscr{L}\left(F_{M}\right)
$$

where $M=(q-1) n^{2}+1$ is an integer.

This is in particular shows that the isomorphism class of $\mathscr{L}\left(F_{q}\right)$ depends neither on the representation $q=(N-1)+\frac{k}{n^{2}}$ (i.e. on the choice of the denominator), nor on the choice of the place of the entries that are removed from $X^{N}$.

A more formalized version of the description before is given by the following definition:

Definition 1. Let $A$ be a $W^{*}$-algebra with faithful trace $\tau$; let $\left(X^{0}, X^{1}, \ldots, X^{n}\right)$ be an (ordered) family of selfadjoint element in $A, n \geqq 2$.

The family $\left(X^{0}, X^{1}, \ldots, X^{n}\right)$ will be incomplete semicircular, if there exists a larger $W^{*}$-algebra $\tilde{A}$ with trace $\tilde{\tau},\left.\tilde{\tau}\right|_{A}=\tau$, and a family $\left(X^{0}, X^{1}, \ldots, X^{n-1}, \tilde{X}^{n}\right)$ which is (free) semicircular in $\tilde{A}$ and such that there exists a partition of the unity $\left(e_{i}\right)_{i=1}^{r}$ in $\left(X^{0}\right)^{\prime \prime}$ with projections of trace $\frac{1}{r}$, such that $X^{n}=\tilde{X}^{n}-\sum_{s=1}^{k} e_{i_{s}} \tilde{X}^{n} e_{j_{s}}$ where $\left(i_{s}, j_{s}\right)_{s=1}^{k}$ are distinct elements in $\{1,2, \ldots, r\}^{2}$. We assume that for each $s=1,2, \ldots, k$, there exists $s^{\prime}$ with $i_{s}=j_{s^{\prime}} ; j_{s}=i_{s^{\prime}}$.

The number $\frac{k}{r^{2}}$ will be called the total area of the holes of the incomplete semicircular family $\left(X^{0}, X^{1}, \ldots, X^{n}\right)$.

In particular this definition and the considerations before make clear the following definition.

Definition 2. Let $q \geqq 2$ be a rational number and assume that $q=N+\frac{k}{r^{2}}$, where $k \in\left\{0,1, \ldots, r^{2}-1\right\}, r \in \mathbb{N}, r \geqq 2$. Let $\left(X^{0}, X^{1}, \ldots, X^{N}\right)$ be an incomplete semicircular family, in a type $I I_{1}$ factor $A$, with total area of the holes $\frac{r^{2}-k}{r^{2}}$. Then the von Neumann algebra generated by $\left(X^{0}, \ldots, X^{N}\right)$ will be a type $I I_{1}$ factor, denoted by $\mathscr{L}\left(F_{q}\right)$ and whose isomorphism class doesn't depend on the choice of the incomplete semicircular family $\left(X^{0}, X^{1}, \ldots, X^{N}\right)$. 
The process of iterating the reduction procedure by rational projections in [4] will let us consider the following ordering relation on incomplete semicircular families.

Definition 3. Let $\left(X^{0}, X^{1}, \ldots, X^{n}\right),\left(Y^{0}, Y^{1}, \ldots, Y^{N}\right)$ be two incomplete semicircular families in a type $I I_{1}$ factor $A$. The family $\left(Y^{0}, Y^{1}, \ldots, Y^{N}\right)$ covers holes of the family $\left(X^{0}, X^{1}, \ldots, X^{N}\right)$ if $Y^{0}=X^{0}, \ldots, Y^{N-1}=X^{N-1}$ and there exists a partition of the unity $\left(e_{i}\right)_{i=1}^{r}$ with projections of trace $\frac{1}{r}$ in $\left(X^{0}\right)^{\prime \prime}$, and distinct elements $\left(i_{s}, j_{s}\right)_{s=1}^{k}$ in $\{1,2, \ldots, r\}^{2}$, such that no one of the entries $e_{i_{s}} Y^{N} e_{j_{s}}, s=1,2, \ldots, k$ contains a hole of $Y^{N}$, and $X^{N}=Y^{N}-\sum_{s} e_{i_{s}} Y^{N} e_{j_{s}}$. (We again assume that for each $s=1,2, \ldots, k$ there exists $s^{\prime}$ in $\{1,2, \ldots, k\}$ with $i_{s}=j_{s^{\prime}}, j_{s}=i_{s^{\prime}}$.)

Moreover in our iterative reduction process, we will have to reduce the algebras with projections that have a special position with respect to the holes of $X^{N}$. This is shown by the following definition.

Definition 4. A projection e covers the holes of the incomplete semicircular family $2\left(X^{0}, X^{1}, \ldots, X^{N}\right)$ if (with the notations introduced in Definition 1),

$$
e \geqq e_{i_{s}}, \quad e \geqq e_{j_{s}}, \quad s=1,2, \ldots, k .
$$

With these definitions, we can now outline the proof of our theorem.

Choose $p / q$ close enough to $\frac{1}{\sqrt{u}}, p / q \geqq \frac{1}{\sqrt{u}}$, so that the integer part of

$$
(N-1) \frac{q^{2}}{p^{2}}+1
$$

is $n=(N-1) u$. Then the algebra $\mathscr{L}\left(F_{N}\right)_{p / q}$ will be described by an incomplete semicircular family $\left(Y^{0}, Y^{1}, \ldots, Y^{n}\right)$ in $e_{1} \mathscr{A} e_{1}$ (where $\mathscr{A}=\mathscr{L}\left(F_{N}\right)$ is generated by the semicircular family $\left\{X^{1}, \ldots, X^{N}\right\}, e_{1} \in\left(X^{1}\right)^{\prime \prime}$ and $\tau\left(e_{1}\right)=p / q$, where $\tau$ is the trace on $\mathscr{A})$.

Moreover choosing $p / q$ close enough to $\frac{1}{\sqrt{u}}$, the area of the holes will be of order $\mathcal{O}(p)$, so if we start with a fixed projection $e$ in $e_{1}\left(X^{1}\right)^{\prime \prime} e_{1}=\left(Y^{0}\right)^{\prime \prime}$ of trace $\tau(e)=\frac{1}{\sqrt{u}}$, then we will be able to find $Y^{n}$ so that there exists a projection $e_{0}$ in $\left(Y^{0}\right)^{\prime \prime}, e \geqq e_{0}$, which covers the holes of $\left(Y^{0}, Y^{1}, \ldots, Y^{n}\right)$.

We choose afterwards a decreasing sequence $\left(e_{k}\right)$ in $\left(Y^{0}\right)^{\prime \prime}$ of rational projections, $e_{k}$, which converges strongly to $e$ and show recursively that the algebra $e_{k} \mathscr{A} e_{k}$ admits a system of generators $\left(Y_{k}^{0}, Y_{k}^{1}, \ldots, Y_{k}^{n}\right)$ that cover holes of the incomplete semicircular family $\left(e_{k} Y_{k-1}^{0} e_{k}, \ldots, e_{k} Y_{k-1}^{n} e_{k}\right)$.

The fact that the total area of the holes of $\left(Y_{k}^{0}, \ldots, Y_{k}^{n}\right)$ tends to zero (which is consequence of the fact that

$$
\mathscr{L}\left(F_{N}\right) p_{q_{k}} \simeq \mathscr{L}\left(F_{(N-1)\left(p_{k} / q_{k}\right)^{-2}+1}\right)
$$

and that $\left.\tau\left(e_{k}\right)=p_{k} / q_{k} \rightarrow \frac{1}{\sqrt{u}}\right)$ implies that the strong operator topology limit 
$Y^{n}=\lim _{k} e Y_{k}^{n} e$ exits and that the family $\left(\tau(e)^{-1 / 2} e Y^{0} e, \cdots, \tau(e)^{-1 / 2} e Y^{n} e\right)$ is (free) semicircular.

Moreover choosing $\tau\left(e_{k}\right)$ to decrease sufficiently fast to $\frac{1}{\sqrt{u}}$, we will be able to prove that elements in any finite set $F \subseteq e \mathscr{A} e$ are very close to the algebra generated by $\left(e_{k} Y_{k}^{0} e_{k}, \ldots, e_{k} Y_{k}^{n} e_{k}\right)$. This will imply that the limit family $\left(e Y^{0} e, \ldots, e Y^{n} e\right)$ generates the reduced algebra.

In finding a family of generators for the reduced algebra, the following lemma (from [4]) will be essential. We restate it in a little more precise form, referring for proof to [4].

Lemma 1. ([4], see also Lemma 3.1 in [7]). Let $\mathscr{A}$ be a $W^{*}$-algebra, generated by a system of selfadjoint elements $\Sigma$ and $\left(w_{i j}\right)_{i, j=1}^{r}$ a matrix unit. Let $k$ be an integer in $\{1,2, \ldots, r\}$ and $e$ be the projection $w_{11}+\cdots+w_{k k}$. Then a system of generators for $e \mathscr{A} e$ is $e \Sigma e,\left(w_{i, j}\right)_{i, j=1}^{k}$ and the set

$$
\Sigma^{\prime}=\bigcup_{\substack{1 \leq i \leq j \leq r \\ j \geqq k+1}}\left\{w_{p(i, j, \alpha), i} \alpha w_{j, q(i, j, \alpha)} \mid \alpha \in \Sigma\right\},
$$

where $p(i, j, \alpha), q(i, j, \alpha)$ are arbitrary functions on $i, j, \alpha$ with values in $\{1,2, \ldots, k\}$.

Remark 1.2. In addition if $1 \leqq k_{-} \leqq k \leqq k_{+}$, and $e_{ \pm}=\sum_{i=1}^{k_{ \pm}} w_{i i}$, then $e_{-} \leqq e \leqq e_{+}$, and if $f=\left(1-e_{+}\right)+e_{-}$, let $B_{f}$ the von Neumann algebra generated in $f \mathscr{A} f$ by the set $f \Sigma f=\{f \alpha f \mid \alpha \in \Sigma\}$ and the matrix unit

$$
\mathscr{W}_{0}=\left\{w_{i j} \mid i, j \in\left\{1,2, \ldots, k_{-}\right\} \cup\left\{k_{+}+1, \ldots, r\right\}\right\} .
$$

If the functions $p, q$ are assumed to take values less than or equal to $k_{-}$, whenever $i \geqq k_{+}+1$, then $e_{-} \Sigma e_{-} \cup e_{-} \Sigma^{\prime} e_{-} \cup\left(w_{i j}\right)_{i, j=1}^{k_{-}}$contains a system of generators for $e_{-} B_{f} e_{-}$.

Proof. This is a corollary of the foregoing lemma applied to the $W^{*}$-algebra $\mathscr{B}_{f}$ generated by $f \Sigma f$ and the matrix unit $\mathscr{W}_{0}$. Q.E.D.

\section{Properties of Incomplete Semicircular Families}

In this paragraph we will state and prove some elementary properties on incomplete semicircular families.

First we will show that the distance in the norm \|\|$_{\tau}$ (given by the trace), between two incomplete semicircular families, one of them covering holes of the other, is an expression of the difference in the total area of the holes. This will be a direct consequence of the normalization conditions imposed on the entries of a semicircular family, and of the fact that these entries are orthogonal with respect to the scalar product given by the trace.

Secondly we will state in a formal way the fact that whenever the total area of the holes of an incomplete semicircular family tends to zero, the family tends to obey the relations governing a (free) semicircular family.

Both these properties will be essential in the limit procedure, when showing that the limit of the sequence of (iterative) incomplete semicircular families is semicircular. 
The last property that we will prove in this paragraph, which is a little bit technical, will concern the way in which, starting from an incomplete semicircular family and a family of entries, one may glue them into another incomplete semicircular family, covering holes of the initial one.

We now state these remarks precisely.

Remark 1. Let $\left(X^{0}, X^{1}, \ldots, X^{n}\right),\left(Y^{0}, Y^{1}, \ldots, Y^{n}\right)$ be incomplete semicircular families in the type $\mathrm{II}_{1}$ factor $\mathscr{A}$ with trace $\tau$, with total area of the holes $\alpha$ and respectively $\beta$. If $\left(\left(Y^{0}, Y^{1}, \ldots, Y^{n}\right)\right.$ covers holes of $\left(X^{0}, X^{1}, \ldots, X^{n}\right)$ then $\left\|Y^{n}-X^{n}\right\|_{\tau}^{2}=\alpha-\beta$.

Proof. Using the representation (Definition 3), of incomplete semicircular families given at the beginning of Sect. 2, we have that this last norm is $\frac{k}{r^{2}}$ (each term of the sum being $\left.\frac{1}{r^{2}}\right)$. But obviously this is exactly the difference in the total area of the holes. Q.E.D.

As we mentioned before, the next remark will show that in an incomplete semicircular family, the value of the traces of the monomials in the family is sufficiently close to the value of the traces of similar monomials in a free semicircular family, if the total area of the holes is small enough.

Remark 2. Let $\left(Z_{n}\right)_{n=1}^{N}$ be a free semicircular family in a $W^{*}$ algebra with trace $\tau$. Let $\mathscr{F}$ be a finite set of monomials in the variables $\left(Z_{n}\right)_{n=1}^{N}$. Then for any $\varepsilon>0$ there exists $\delta_{\varepsilon}$, such that for any incomplete semicircular family $\left(X^{1}, \ldots, X^{N}\right)$, with total area of the holes less than $\delta_{\varepsilon}$, for each $m$ in $\mathscr{F}$, if $\phi(m)$ is the corresponding monomial in the variables $\left(X^{1}, \ldots, X^{N}\right)$, then $|\tau(m)-\varphi(\phi(m))| \leqq \varepsilon$, for any $m \in \mathscr{F}$. Here $\varphi$ is the (normalized) trace on the $\mathrm{II}_{1}$ factor generated by $\left(X^{1}, \ldots, X^{N}\right)$.

Proof. By the unicity (up to isomorphism) of a free semicircular family, we may assume that $\left(Z^{1}, \ldots, Z^{N}\right)$ is exactly the free semicircular family $X^{1}, \ldots, X^{N-1}$, $\tilde{X}^{N}$ covering the holes of $X^{1}, \ldots, X^{N}$ (Definition 1). The proof then follows from the preceding remark, with a trivial limit argument. Q.E.D.

By Voiculescu's theorem, any incomplete semicircular family $\left(X^{0}, \ldots, X^{n}\right)$ appears as follows: take $D$ be a type $\mathrm{II}_{1}$ factor with trace $\tau$, let $\left(e_{i j}\right)_{i, j=1}^{r}$ be the matrix unit of $M_{r}(\mathbb{C})$ and consider in $D \otimes M_{r}(\mathbb{C})$,

$$
X^{s}=r^{-1 / 2}\left(\sum_{i=1}^{r} f(i, s) \otimes e_{i i}+\sum_{1 \leqq i<j \leqq r} g(i, j, s) \otimes e_{i j}+g(i, j, s)^{*} \otimes e_{j i}\right),
$$

where $s=1,2, \ldots, n$, and where for $s=n$ some of the $f(i, n)$ or $g(i, j, n)$ might be zero.

Further let $a$ be a selfadjoint semicircular element in $D$, let $A$ be the abelian von Neumann subalgebra of $D \otimes M_{r}(\mathbb{C})$ generated by $\left\{a \otimes e_{i i}\right\}_{i=1}^{r}$. Assume further that the family $\omega_{1}$ of nonnull entries of the form

$$
\{f(i, s) \mid s=1,2, \ldots, n, i=1, \ldots, r\}
$$

is semicircular in $D$, and that the family $\omega_{2}$ of the nonzero elements

$$
\{g(i, j, s) \mid 1 \leqq i<j \leqq r, s=1,2, \ldots, n\}
$$


is circular.

Moreover assume that $\omega_{1} \cup \omega_{2} \cup\{a\}$ is a free family of elements in $D$. If $X^{0}$ is any semicircular element generating $A$, then by Voiculescu's theorem $(2.8,[7])$ the family $\left(X^{0}, X^{1}, \ldots, X^{n}\right)$ is incomplete semicircular, and conversely, any incomplete semicircular family appears in this way.

In the next lemma, starting from a von Neumann algebra $\mathscr{A}$ generated by both an incomplete semicircular family as before and a number $k$ of entries of the form $f \otimes e_{p p}$ or $g \otimes e_{p q}$, which still obey the freeness conditions together with the entries of $\left(X^{s}\right)_{s=1}^{n}$, ( $k$ is less than the number of holes of $\left.\left(X^{s}\right)_{s=1}^{n}\right)$, we construct an incomplete semicircular family covering holes of $\left(X^{0}, \ldots, X^{n}\right)_{s=1}^{n}$.

Lemma 3. Assume that $X^{0}, X^{1}, \ldots, X^{n}, n \geqq 2$ is an incomplete semicircular family, described as before in $D \otimes M_{r}(\mathbb{C})$ and suppose that in addition we are given families

$$
C_{s}=\left\{f_{t}^{\prime} \otimes e_{p_{t}^{\prime} p_{t}^{\prime}} \mid t=1,2, \ldots, d\right\} \subseteq D \otimes M_{r}(\mathbb{C})
$$

and

$$
C_{c}=\left\{g_{s}^{\prime} \otimes e_{p_{s} p_{s}} \mid s=1,2, \ldots, b\right\} \subseteq D \otimes M_{r}(\mathbb{C}),
$$

where $p_{s} \neq q_{s}, p_{s}, q_{s}, p_{t}^{\prime} \in\{1,2, \ldots, r\}, t=1, \ldots, d ; s=1, \ldots, b$. Let

$$
\bar{C}_{s}=\left\{f_{t}^{\prime} \mid t=1,2, \ldots, d\right\}
$$

and

$$
\bar{C}_{c}=\left\{g_{s}^{\prime} \mid s=1,2, \ldots, b\right\} .
$$

Assume that the family $\Omega_{1}=\omega_{1} \cup \bar{C}_{s}$ is semicircular; that the family $\Omega_{2}=\omega_{2} \cup \bar{C}_{c}$ is circular and that the family $\Omega_{1} \cup \Omega_{2} \cup\{a\}$ is free in $D$. Moreover if $k$ is the number of holes of the family $\left(X^{0}, X^{1}, \ldots, X^{n}\right)$ in this description (or what is the same, if the total area of holes is $k / r^{2}$ ), we assume that $d+2 b \leqq k$ and that $d$ is even.

Then we may find an incomplete semicircular family $\left(X^{0}, \ldots, X^{n-1}, Y^{n}\right)$, which covers holes of the initial family $\left(X^{0}, X^{1}, \ldots, X^{n}\right)$ and such that the von Neumann algebra $\mathscr{A}$ generated by $\left(X^{0}, X^{1}, \ldots, X^{n}\right) \cup C_{s} \cup C_{c}$ coincides with the one generated by $\left(X^{0}, \ldots, X^{n-1}, Y^{n}\right)$.

Proof. Let $\left(v(1, p, 1) \otimes e_{1}\right)\left(b_{1 p} \otimes e_{p p}\right)$ (as in Theorem 3.3 [7]) be the polar decomposition (in $D$ ) of $g(1, p, 1) \otimes e_{1 p}, p=2, \ldots, r$. (in $D$ ).

Let $\left(w_{i j}\right)_{i, j=1}^{r}$ be the matrix unit in $\mathscr{A}$, obtained from the partial isometries

$$
w_{i j}=\left[v(1, i, 1)^{*} v(1, j, 1)\right] \otimes e_{i j}, \quad i, j=1,2, \ldots, r,
$$

where $v(1,1,1)=1$. The following changes labeled from 1) to 4$)$ in the structure of the sets $C_{c}, C_{s}$ and $\Omega_{1}, \Omega_{2}$, will give new sets, $C_{c}^{\prime}, C_{s}^{\prime}, \Omega_{1}^{\prime}, \Omega_{2}^{\prime}$, with the same properties as those of the initial ones: $\Omega_{1}^{\prime}$ is semicircular, $\Omega_{2}^{\prime}$ is circular with $\Omega_{1}^{\prime} \cup \Omega_{2}^{\prime} \cup\{a\}$ free and $\mathscr{A}$ is generated by $\left(X_{s}\right)_{s=0}^{n}$ and $C_{c}^{\prime}, C_{s}^{\prime}$.

1) Removing from $C_{s}$ two elements $f_{t}^{\prime} \otimes e_{p_{t}^{\prime} p_{t}^{\prime}} f_{s}^{\prime} \otimes e_{p_{s}^{\prime} p_{s}^{\prime}} t, s \in\{1,2, \ldots, d\}, t \neq s$, with $p_{t}^{\prime}=p_{s}^{\prime}$, and adding the element

$$
w_{p_{0} p_{t}^{\prime}}\left[\frac{f_{t}^{\prime}}{\sqrt{2}} \otimes e_{p_{t}^{\prime} p_{t}^{\prime}}+i \frac{f_{s}^{\prime}}{\sqrt{2}} \otimes e_{p_{s}^{\prime} p_{s}^{\prime}}\right]
$$

to the set $C_{c}$, where $p_{0} \neq p_{t}^{\prime}, p_{0} \in\{1,2, \ldots, r\}$. 
2) Removing an element $g_{s}^{\prime} \otimes e_{p_{s} q_{s}}, s=1,2, \ldots, b$ from the set $C_{c}$, and adding to $C_{s}$ the real and imaginary part of

$$
\sqrt{2}\left[g_{s}^{\prime} \otimes e_{p_{s} q_{s}}\right] w_{q_{s} q_{s}} .
$$

3) Take an element $f_{s}^{\prime} \otimes e_{p_{s} q_{s}}$ from $C_{s}$ and replace it, in $C_{s}$, by

$$
w_{p p_{t}^{\prime}}\left[f_{s}^{\prime} \otimes e_{p_{s}^{\prime} p_{s}^{\prime}}\right] w_{p_{s}^{\prime} p},
$$

where $p_{s}^{\prime} \neq p, p \in\{1,2, \ldots, r\}$.

4) Take $g_{s}^{\prime} \otimes e_{p_{s} q_{s}}$ in $C_{c}$ and replace it in $C_{c}$ by

$$
w_{p p_{s}}\left[g_{s}^{\prime} \otimes e_{p_{s} q_{s}}\right] w_{q_{s} q},
$$

where $p \neq q, p, q \in\{1,2, \ldots, r\}$.

It is clear that a repeated application of the steps before will enable us to assume that the elements in the new sets $C_{s}^{\prime}, C_{c}^{\prime}$ are in one to one correspondence with some of the vanishing entries of $X^{n}$ (since $d+2 b \leqq k$ ). Hence we may take

$$
Y^{n}=X^{n}+\sum_{\alpha \in C_{s}} \frac{\alpha}{\sqrt{r}}+\sum_{\beta \in C_{c}}\left(\frac{\beta+\beta^{*}}{\sqrt{r}}\right) .
$$

Since the hypothesis on the families of the entries in $\Omega_{1}^{\prime}, \Omega_{2}^{\prime}$ are still fulfilled (by the next lemma), it follows that $\left(X^{0}, X^{1}, \ldots, X^{n-1}, Y^{n}\right)$ is the required family.

Q.E.D.

The next lemma gives the reasons for which the elements in the new family $\Omega_{1}^{\prime}$, $\Omega_{2}^{\prime}$ have the property that $\Omega_{1}^{\prime}$ is semicircular, $\Omega_{2}^{\prime}$ is circular, while $\Omega_{1}^{\prime} \cup \Omega_{2}^{\prime} \cup\{a\}$ is free.

Lemma 4. Suppose that $D$ is a $W^{*}$-algebra with trace $\tau$ and that $\omega_{1}=\left\{f_{i}\right\}_{i \in I}$ is a free semicircular family, $\omega_{2}=\left\{g_{j}\right\}_{j \in J} \cup\left\{g_{k}^{\prime}\right\}_{k \in K}$ is a free circular family and that $\omega_{1} \cup \omega_{2}$ is free.

Let $\left\{v_{k}\right\}_{k \in K}$ be the unitaries from the polar decomposition of the elements in $\left\{g_{k}^{\prime}\right\}_{k \in K}$. Then each of the following changes in $\omega_{1}, \omega_{2}$ will let us obtain new sets $\omega_{1}^{\prime}$, $\omega_{2}^{\prime}$ in $D$, such that $\omega_{1}^{\prime}$ is still semicircular, $\omega_{2}^{\prime}$ is circular, while $\omega_{1}^{\prime} \cup \omega_{2}^{\prime}$ is free:

a) Remove distinct $\left(f_{1}, f_{2}\right)$ from $\omega_{1}$ and add $\frac{f_{1}}{\sqrt{2}}+\frac{i f_{2}}{\sqrt{2}}$ at $\omega_{2}$;

b) Remove $g$ from $\omega_{1}$ and add $\sqrt{2} \operatorname{Re} g, \sqrt{2} \operatorname{Im} g$ to $\omega_{1}$;

c) Replace $f$ in $\omega_{1}$ by $w f w^{*}$, where $\omega$ is a product in the elements $\left\{v_{j}\right\}_{j \in K}$;

d) Replace $g$ in $\omega_{2}$ by $w_{1} g w_{2}$ where $w_{1}, w_{2}$ are products (eventually void) in the elements $\left\{v_{k}\right\}_{k \in K}$.

Proof. By Proposition 2.6 in [7], an element $g$ is circular if and only if the elements coming from its polar decomposition $g=v b$, are a free family $\{v, b\}$, and $\tau\left(v^{k}\right)$ is $\delta_{k 0}$ (the Kronecker symbol) for $k \in \mathbb{Z}$, while

$$
\tau\left(b^{k}\right)=\frac{4}{\pi} \int_{0}^{1} t^{k}\left(1-t^{2}\right)^{1 / 2} d t, \quad k \in \mathbb{N} .
$$

For each such a positive element $b=b(g)$, let $B(g)$ be an assignment of an unitary in $D$ such that $B(g)^{\prime \prime}=(b(g))^{\prime \prime}$ and $\tau\left((B(g))^{k}\right)=\delta_{k 0}, k \in \mathbb{Z}$, (the Lebesgue distribution). 
In these terms $g=v(g) b(g)$ is circular if and only if the family $\{v(g), B(g)\}$ is free, while $v(g)$ and $B(g)$ have the Lebesgue distribution.

Likewise for a semicircular element $f$, we assign an unitary $F=F(f)$ with the Lebesgue distribution, so that $(F(f))^{\prime \prime}=\{f\}^{\prime \prime}$. In particular a family $C_{s}$ in $D$ is semicircular if and only if $\left\{F(f) \mid f \in C_{s}\right\}$ is free.

Moreover note that if $g$ is any element in $D$, and $w_{1}, w_{2}$ are unitaries in $D$, then the polar decomposition of $w_{1} v w_{2}$ is $\left(w_{1} g w_{2}\right)\left(w_{2}^{*} b w_{2}\right)$, if $g=v b$, so that we may assume that

$$
\begin{aligned}
& v\left(w_{1} g w_{2}\right)=w_{1} v(g) w_{2}, \\
& B\left(w_{1} g w_{2}\right)=w_{2}^{*} B(g) w_{2} .
\end{aligned}
$$

Also we may assume that $F\left(w f w^{*}\right)=w F(f) w^{*}$ for $f$ in $C_{s}, w$ unitary.

Hence to prove the validity of steps, $c, d$ we have to prove that the families

$$
\left\{w F\left(f_{0}\right) w^{*}\right\} \cup\{F(f)\}_{f \in \omega_{1} /\left\{f_{0}\right\}} \cup\left\{v\left(g_{j}\right), B\left(g_{j}\right)\right\}_{j \in J} \cup\left\{v\left(g_{k}^{\prime}\right), B\left(g_{k}^{\prime}\right)\right\}_{k \in K}
$$

and

$$
\begin{gathered}
\{F(f)\}_{f \in \omega_{1}} \cup\left\{w_{1} v\left(g_{j_{0}}\right) w_{2}\right\} \cup\left\{w_{1}^{*} B\left(g_{j_{0}}\right) w_{1}\right\} \\
\cup\left\{v\left(g_{j}\right), B\left(g_{j}\right)\right\}_{j \in J \backslash\left\{j_{0}\right\}} \cup\left\{v\left(g_{k}^{\prime}\right), B\left(g_{k}^{\prime}\right)\right\}_{k \in K}
\end{gathered}
$$

are free if $w, w_{1}, w_{2}$ are products in the elements $\left\{v\left(g_{k}^{\prime}\right) \mid k \in K\right\}$.

Here we know that the family

$$
\left.\{F(f)\}_{f \in \omega_{1}} \cup\left\{v\left(g_{j}\right)\right\}, B\left(g_{j}\right)\right\}_{j \in J} \cup\left\{v\left(g_{k}^{\prime}\right), B\left(g_{k}^{\prime}\right)\right\}_{k \in K}
$$

is free. The freeness of the two families comes from the following elementary fact concerning free groups: If $\left\{x, y_{1}, \ldots, y_{N}\right\}$ are the generators of a free group $F_{N+1}$ then so are

$$
\left\{x y_{1}, y_{1}, \ldots, y_{N-1}, y_{N}\right\} \text {. }
$$

This proves the steps c) and d). The steps a), b) are simply the definition of a circular element. Q.E.D.

\section{Technical Result}

In this paragraph we prove a technical result which is an adaptation of the technical Lemma 2 in [4]. In that paper we proved that whenever $\left(X^{s}\right)_{s \in S}$ is a free semicircular family, generating the von Neumann algebra $\mathscr{A} \simeq \mathscr{L}\left(F_{\infty}\right)$ and $e$ is any projection in the von Neumann algebra $\left\{X^{\sigma}\right\}^{\prime \prime}$ of one of the generators $X^{\sigma}$, then the family $\left\{e X^{s} e\right\}_{s \in S}$ is contained in a larger semicircular family $\left(Y_{t}\right)_{t \in T}$ that still generates $e A e$.

As in the proof of that lemma, the main point is to prove this result first for $e$ of rational trace, but to add in this case certain requirements that make a limit procedure possible. In our case we will start with an incomplete semicircular family $X^{0}, X^{1}, \ldots, X^{n}$ that generates $\mathscr{A}=\mathscr{L}\left(F_{r}\right)$ (where $r \in \mathbb{Q} \cap[2, \infty), n=[r]$ ). We assume that the total area of the holes of this family is small enough so that there exists a non-trivial projection $e_{0}$ in $\left\{X^{0}\right\}^{\prime \prime}$ covering the holes of the family (see Definition 3).

Then we start with a projection $e_{1} \geqq e_{0}$, of rational trace $p / q$, which is close enough to the identity so that the integer part of the numbers of generators that are 
needed for the reduced algebra

$$
e_{1} \mathscr{A} e_{1} \cong \mathscr{L}\left(F_{(r-1)(p / q)^{-2}-1}\right),
$$

does not increase (i.e. is equal to $n$ ).

Applying the lemma at the end of Sect. 2 that describes the form of the generators for the reduced algebra, and then gluing these generators together, by the procedure described in Lemma 3 , we will be able to find a family $\left(e_{1} X^{0} e_{1}, \ldots, e_{1} X^{n-1} e_{1}, Y^{n}\right)$, that covers holes of the family $\left(e_{1} X^{i} e_{1}\right)_{i=0}^{n}$ and generates the reduced algebra $e_{1} \mathscr{A} e_{1}$. Thus the new pieces of generators coming from the band determined by the projection $\left(1-e_{1}\right)$ in $\mathscr{A}$ are filling the holes of $e_{1} X^{n} e_{1}$.

The precise statement of the procedure described before is the following.

Lemma 5. Let $\left(X^{0}, X^{1}, \ldots, X^{n}\right), n \geqq 2$ be an incomplete semicircular family with total area of the holes $\alpha \in(0,1) \cap Q$, generating the von Neumann algebra

$$
\mathscr{A} \simeq \mathscr{L}\left(F_{n+1-\alpha}\right)
$$

with trace $\tau$.
$\quad$ For any projection $e$ in $\left(X_{0}\right)^{\prime \prime}$ of rational $t=\tau(e) \geqq\left(1-\frac{\alpha}{n}\right)^{1 / 2}$, which covers the holes of $X^{n}$, (see Definition 4), there exists a semicircular incomplete family

$$
\left(\tau(e)^{-1 / 2} e X^{0} e, \ldots, \tau(e)^{-1 / 2} e X^{n-1} e, \tau(e)^{-1 / 2} Y^{n}\right)
$$

which covers holes of $\left(\tau(e)^{-1 / 2} e X^{i} e\right)_{i=0}^{n}$ and generates e⿻A $e$.

In addition the total area of the holes of this new family (with respect to the

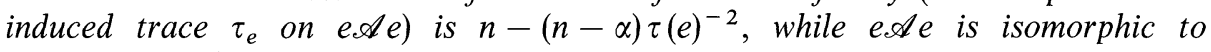
$\mathscr{L}\left(F_{(n-\alpha) t^{-2}+1}\right)$.

Out of the proof of Lemma 5, the following corollary will come immediately.

Corollary 6. In addition if $e_{-} \leqq e$ is any other projection in $\left\{X^{0}\right\}^{\prime \prime}$, of rational trace and covering the holes of $\left(X^{0}, X^{1}, \ldots, X^{n}\right)$ then

$$
e_{-} B e_{-} \subseteq\left\{\left(e_{-} X^{i} e_{-}\right)_{i=0}^{n-1}, e_{-} Y^{n} e_{-}\right\}^{\prime \prime},
$$

where $B=B_{f}$ is the von Neumann algebra generated in $f \mathscr{A} f$ by the elements $\left\{f X^{i} f \mid i=0, \ldots, n\right\}$ with $f=(1-e)+e_{-}$.

Proof of Lemma 5. Assume that $1-\alpha=\frac{k}{r^{2}}, \tau(e)=\frac{p}{r}$, where $p, k, r \in \mathbb{N}, k \leqq r^{2}-1$ and that $X^{0}, X^{1}, \ldots, X^{n}$ has the representation below, in $D \otimes M_{r}(\mathbb{C})$, where $D$ is a type $\mathrm{II}_{1}$ factor with trace $\varphi$, containing an infinite free family.

Assume that $\left(e_{i j}\right)_{i, j=1}^{r}$ is the matrix unit of $M_{r}(\mathbb{C})$ and let

$$
\left\{X^{0}\right\}^{\prime \prime}=\left\{a \otimes e_{i i} \mid 1 \leqq i \leqq r\right\}^{\prime \prime}
$$

and

$$
\left.X^{s}=r^{-1 / 2}\left(\sum_{i=1}^{r} f(i, s) \otimes e_{i i}+\sum_{1 \leqq i<j \leqq r}(g(i, j, s)) \otimes e_{i j}+g(i, j, s)^{*} \otimes e_{j i}\right)\right),
$$

$s=1,2, \ldots, n$.

When $s=n, k^{\prime}$ of the diagonal entries $f(i, n)$ are vanishing, while $k^{\prime \prime}$ of the elements $\{g(i, j, n) \mid 1 \leqq i<j \leqq n\}$ are null and $k^{\prime}+2 k^{\prime \prime}=r^{2}-k$. 
In addition the collection of all nonzero elements

$$
\omega_{2}=\{g(i, j, s) \mid 1 \leqq i<j \leqq r, s=1,2, \ldots, n\}
$$

form a free circular family, while

$$
\omega_{1}=\{f(i, s) \mid 1 \leqq i \leqq r, s=1,2, \ldots, n, f(i, s) \neq 0\}
$$

is a free circular family. Moreover $\omega_{1} \cup \omega_{2} \cup\{a\}$ is a free family in $D$.

Moreover such a representation is possible with the supplementary condition $e=\sum_{1 \leqq i \leqq p} 1 \otimes e_{i i}$. Since $e$ covers the holes of $X^{n}$ it follows that for $j \geqq p+1$, no $g(i, j, n), 1 \leqq i<j \leqq r$, or $f(j, n)$ vanishes.

We take as generator for the algebra $\left\{X^{0}\right\}^{\prime \prime}$ the element $\sum_{t=1}^{r}(a+2 t) \otimes e_{t t}$ and we apply Lemma 1 to obtain a system of generators for the reduced algebra $e \mathscr{A} e$. Here $\mathscr{A}$ is generated by $\left(X^{0}, X^{1}, \ldots, X^{n}\right)$.

To get a matrix unit we take as in [7], the polar decomposition

$$
g(1, t, 1) \otimes e_{1 t}=\left[v(1, t) \otimes e_{1 t}\right]\left(b(1, t) \otimes e_{t t}\right),
$$

$t=2, \ldots, r$, let $v(1,1)=1$ and

$$
w_{i j}=v(1, i)^{*} v(1, j), \quad 1 \leqq i, j \leqq r .
$$

Hence by Lemma 1 , we have that a system of generators for $e \mathscr{A} e$ is $\left(e X^{i} e\right)_{i=0}^{n}$, $\left(w_{i j} \otimes e_{i j}\right)_{i, j=1}^{p}$ (which is already contained in $\left.\left\{\left(e X^{i} e\right)_{i=0}^{n}\right\}^{\prime \prime}\right)$ and the following sets of elements:

$$
\begin{aligned}
A & =\left\{w_{p_{i} i} a w_{i p_{i}} \otimes e_{p_{i} p_{i}} \mid p+1 \leqq i \leqq r\right\}, \\
B & =\left\{w_{p(i, s), i} f(i, s) w_{i, p(i, s)} \otimes e_{p(i, s), p(i, s)} \mid p+1 \leqq i \leqq r, s, s=1, \ldots, n\right\}, \\
C & \left.=w_{q(i, j, s), i} g(i, j, s) w_{i, p(i, j, s)} \otimes e_{q(i, j, s), p(i, j, s)} \mid s=2, \ldots, n ; 1 \leqq i<j \leqq r, j \geqq p+1\right\}, \\
D & =\left\{w_{q(i, j), i} g(i, j, 1) w_{j, p(i, j)} \otimes e_{q(i, j), p(i, j)} \mid 2 \leqq i<j \leqq r, j \geqq p+1\right\}, \\
E & =\left\{w_{p(i), i} b(1, i) w_{i, p(i)} \otimes e_{p(i), p(i)} \mid p+1 \leqq i \leqq r\right\} .
\end{aligned}
$$

Note that in the sets $B$ and $D$, none of the listed elements vanishes since by hypothesis the holes of $X^{n}$ are covered by the projection $e$. Here

$$
p_{i} ; p(i, s) ; p(i, j, s) \neq q(i, j, s) ; p(i, j) \neq q(i, j), p(i)
$$

are arbitrary functions on the variables $i, j, s$ taking values into the set $\{1,2, \ldots, p\}$. The next lemma (Lemma 7) shows that the family $\omega_{1}^{\prime}$ of the diagonal entries of $\left(e X^{s} e\right)_{s=0}^{n}$ union with the sets $A, B, E$ and $\{a\}$ is free semicircular, while the family of the upper diagonal entries of $\left(e X^{s} e\right)_{s=0}^{n}$ union with the sets in $C, D$ is circular, while the family $\omega_{1}^{\prime} \cup \omega_{2}^{\prime}$ is free. Hence the conditions in Lemma 3 are fulfilled if we can show that

$$
\operatorname{card}(A \cup B \cup E)+2 \operatorname{card}(C \cup D)<r^{2}-k .
$$

Here $r^{2}-k$ is the number of holes of the incomplete semicircular family

$$
X^{0}, X^{1}, \ldots, X^{n}
$$

in its representation in $D \otimes M_{r}(\mathbb{C})\left(\right.$ as $\left(\frac{k}{r^{2}}\right)=1-\alpha$, where $\alpha$ is the total area of the holes). Moreover, we may always assume that $r-p$ is even; this will insure that $\operatorname{card}(A \cup B \cup E)$ is even, which is one of the conditions in Lemma 4 . 
Indeed it is obvious that

$$
d=\operatorname{card}(A \cup B \cup E)=(n+2)(r-p),
$$

while

$$
\text { card } C=\frac{1}{2}(n-1)\left[\left(r^{2}-r\right)-\left(p^{2}-p\right)\right]
$$

and

$$
\operatorname{card} D=\frac{1}{2}\left[\left(r^{2}-r\right)-\left(p^{2}-p\right)-(r-p)\right]
$$

so that

$$
2 b=2 \operatorname{card}(C \cup D)=\left[\left(r^{2}-r\right)-\left(p^{2}-p\right)\right]-2(r-p),
$$

and hence

$$
d+2 b=n\left(r^{2}-p^{2}\right) \text {. }
$$

But by hypothesis $\tau(e)>\left(1-\frac{\alpha}{n}\right)^{1 / 2}$ and $\alpha=1-\frac{k}{r^{2}}, \tau(e)=\frac{p}{r}$ so that we get

$$
\left(\frac{p}{r}\right)^{2}>1-\frac{r^{2}-k}{r^{2} n}
$$

which is equivalent to

$$
\frac{r^{2}-k}{n}>\left(n^{2}-p^{2}\right)
$$

In this way we get that $d+2 b<r^{2}-k$ and hence, by Lemma 3, we get an incomplete semicircular family (in $\left.D \otimes M_{p}(\mathbb{C})\right), \quad\left(c^{-1 / 2} e X^{0} e, \ldots\right.$, $\left.c^{-1 / 2} e X^{n-1} e, c^{-1 / 2} Y^{n}\right)$ that covers holes of the reduced family $\left(c^{-1 / 2} e X^{i} e\right)_{i=0}^{n}$, and that generates e⿻Ade. (Here $c=\tau(e)$.)

Moreover out of this representation we get that the number of holes (in the representation in $\left.D \otimes M_{r}(\mathbb{C})\right)$ is

$$
\left(r^{2}-k\right)-n\left(r^{2}-p^{2}\right),
$$

so that the total area of the holes of the new family is

$$
\begin{aligned}
\frac{1}{p^{2}}\left[\left(r^{2}-k\right)-n\left(r^{2}-p^{2}\right)\right] & =\frac{1}{p^{2}}\left[r^{2} \alpha-n\left(r^{2}-p^{2}\right)\right] \\
& =\tau(e)^{-2}(\alpha-n)+n=n-(n-\alpha) \tau(e)^{-2} .
\end{aligned}
$$

In particular, with $t=\tau(e)$ the new family $\left(e X^{0} e, \ldots, e X^{n-1} e, Y^{n}\right)$ generates a von Neumann algebra which is isomorphic to $\mathscr{L}\left(F_{r}\right)$ with

$$
r=(n+1)-n+(n-\alpha) t^{-2}=1+(n-\alpha) t^{-2} .
$$

Hence $e \mathscr{A} e \cong \mathscr{L}\left(F_{(n-\alpha) t^{-2}+1}\right)$ while $\mathscr{A} \cong \mathscr{L}\left(F_{n+1-\alpha}\right)$. This ends the proofs of Lemma 5. Q.E.D.

Proof of Corollary 6. We may assume, taking $r$ big enough, that in the representation described in the proof

$$
\tau\left(e_{-}\right)=\frac{p-}{r}, \quad p_{-} \in \mathbb{N},
$$


and that

$$
e_{-}=1 \otimes e_{1,1}+\cdots+1 \otimes e_{p_{-}, p_{-}} .
$$

Moreover in this case the holes of $\left(X^{0}, X^{1}, \ldots, X^{n}\right)$ are also covered by the projection $e_{-} \leqq e$. Hence if we assume that the functions in formula (1) take values in the set $\left\{1,2, \ldots, p_{-}\right)$, then it will follow by the remark after Lemma 1 (with $\left.e_{+}=e\right)$ that the elements $\left(e_{-} X^{s} e_{-}\right)_{s=0}^{n}$ (which already contain $\left(w_{i j}\right)_{i, j=1}^{p_{-}}$together with the sets $\mathrm{A}, \mathrm{B}, \mathrm{C}, \mathrm{D}, \mathrm{E}$ (which are thus unchanged when they are multiplied with $\left.e_{-}\right)$are still generators for the reduced algebra $e_{-} B_{f} e_{-}$.

The moves in Lemma 3, do not alter this generating property and hence Corollary 6 follows. Q.E.D.

The following lemma, which appeared in the proof of Lemma 5, states that the entries which appear in the reduction process (the sets $A, \ldots, E$ ) give free semicircular (respectively circular) families. These families are also free with the remaining entries of $\left(e_{-} X^{s} e_{-}\right)_{s=0}^{n}$. The set $\left(g_{j}\right)_{j \in K}$ corresponds to $\{g(1, j, 1) \mid 1 \leqq j \leqq p\}$, the set $\left(g_{j}\right)_{j \in L}$ corresponds to $\{g(1, j, 1) \mid r \geqq j \geqq p+1\}$ and $\left(g_{j}\right)_{j \in J}$ corresponds to the remaining $\{g(i, j, s)\}$.

Lemma 7. Let $D$ be a type $I I_{1}$ factor with trace $\tau, \omega_{1}=\left\{f_{i}\right\}_{i \in I} \cup\{a\}$ a free semicircular family, $\omega_{2}=\left\{g_{j}\right\}_{j \in J \cup K \cup L}$. Assume that $\omega_{1} \cup \omega_{2}\{a\}$ is free. Let

$$
\omega_{1}^{\prime}=\left\{w_{i}^{*} f_{i} w_{i}\right\}_{i \in I} \cup\left\{v_{j}^{\prime} a v_{j}^{\prime *}\right\}_{j \in L} \cup\left\{w_{j}^{\prime} b_{j}\left(w_{j}^{\prime}\right)^{*}\right\}_{j \in L} \cup\{a\}
$$

and

$$
\omega_{2}^{\prime}=\left\{w_{j}^{\prime \prime} g_{j} w_{j}^{\prime \prime \prime}\right\}_{j \in J \cup K} .
$$

Here $\left(w_{i}\right)_{i \in I},\left\{w_{j}^{\prime}\right\}_{j \in L}\left\{w_{j}^{\prime \prime}, w_{j}^{\prime \prime \prime}\right\}_{j \in J \cup K}$ are (eventually void) products in the elements $\left\{v_{j}\right\}_{j \in K \cup L}$, while $v_{j}^{\prime}, j \in L$ is such a product that contains the element $v_{j}$. in $D$.

Then $\omega_{1}^{\prime}$ is a semicircular family, $\omega_{2}^{\prime}$ is a circular family, while $\omega_{1}^{\prime} \cup \omega_{2}^{\prime}$ is free

Proof. In the setting of the proof of Lemma 4, we have to show that the union of the families

$$
\left\{w_{i} F\left(f_{i}\right) w_{i}^{*}\right\}_{i \in I} \cup\left\{v_{j}^{\prime} F(a) v_{j}^{\prime *}\right\}_{j \in L} \cup\{F(a)\} \cup\left\{w_{j}^{\prime} F\left(b_{j}\right)\left(w_{j}^{\prime}\right)^{*}\right\}_{j \in L}
$$

and

$$
\left\{w_{j}^{\prime \prime} v\left(g_{j}\right) w_{j}^{\prime \prime \prime} ;\left(w_{j}^{\prime \prime \prime}\right)^{*} B\left(g_{j}\right) w_{j}^{\prime \prime \prime} \mid j \in J \cup K\right\}
$$

is a free family of unitaries with the Lebesgue distribution, if the family

$$
\left\{F\left(f_{i}\right)\right\}_{i \in I} \cup\{F(a)\} \cup\left\{v\left(g_{i}\right) ; B\left(g_{j}\right)\right\}_{j \in K \cup J \cup L}
$$

is a free family of unitaries having the Lebesgue distribution. But this is a consequence of the following two facts concerning free groups:

(i) If $\left\{x_{1}, x_{2}, \ldots, x_{n}, y_{1}, \ldots, y_{k}, a\right\}$ are the generates of $F_{n+k+1}$ then

$$
\left\{x_{i} a x_{i}^{-1}\right\}_{i=1}^{n} \cup\{a\} \cup\left\{y_{j}\right\}_{j=1}^{k}
$$

are free generators of a free group.

(ii) If $\left(x_{1}, \ldots, x_{n}, y\right\}$ is a free system of generators of $F_{n+1}$, then so is

$$
\left\{x_{1} y, x_{2}, \ldots, x_{n}, y\right\} \text {. }
$$

This ends the proof of Lemma 7. Q.E.D. 
Corollary 8. Let $q \geqq 2$ be a rational number, $t$ a rational number in $(0,1)$. Then with the notations of Definition 2,

$$
\mathscr{L}\left(F_{q}\right)_{t} \cong \mathscr{L}\left(F_{(q-1) t^{-2}+1}\right) .
$$

Proof. Let $\left(X^{0}, \ldots, X^{N}\right)$ be an incomplete semicircular family generating $\mathscr{A}=\mathscr{L}\left(F_{q}\right)$ (with $N=$ the integer part of $q$ ) and let $e$ be a projection in $\left(X^{0}\right)^{\prime \prime}$ with trace value $t$. Also let $M$ be the integer part of $(q-1) t^{-2}+1$. If $M=N$ then there exists an incomplete semicircular family $\left(c Z^{0}, \ldots, c Z^{M}\right)$ in e⿻A $e$ with $Z^{i}=e X^{i} e$, $i=0,1, \ldots, N-1, c=t^{-1 / 2}$ that generates $e \mathscr{A} e$ and that covers holes of $\left(e X^{0} e, \ldots, e X^{N} e\right)$.

If $M>N$ then there exists an incomplete semicircular family $\left(c Z^{0}, \ldots, c Z^{M}\right)$ in $e \mathscr{A} e$ that also generates $e \mathscr{A} e$. Moreover if $e_{0}$ is a projection in $e\left(X^{0}\right)^{\prime \prime} \mathrm{e}=\left(Z^{0}\right)^{\prime \prime}$, of rational normalized trace (relative to $e \mathscr{A} e$ ), greater than the square root of the total area of the holes $\left(c Z^{0}, \ldots, c Z^{M}\right)$ (which is $\left.M-(q-1) t^{-2}\right)$, then we may find the incomplete semicircular family $\left(c Z^{0}, \ldots, c Z^{M}\right)$ so that its holes are covered by $e_{0}$.

The case $M>N$ is simply a consequence of Lemma 1 and Lemma 7. The case $M=N$ is simply Lemma 5 .

\section{Proof of the Main Results}

In this paragraph we will prove that $\mathscr{L}\left(F_{N}\right)_{\frac{1}{\sqrt{k}}} \cong \mathscr{L}\left(F_{(N-1) k+1}\right), k, N \in \mathbb{N}, N \geqq 2$. As a consequence we will obtain the stable isomorphism of the von Neumann algebras associated to free groups.

The idea to prove the isomorphism before, is to start with a sequence of rational numbers $\frac{p_{j}}{r_{j}}$ decreasing to $\frac{1}{\sqrt{k}}$, where $\frac{p_{1}}{r_{1}}$ is close enough to $\frac{1}{\sqrt{k}}$ so that the first reduction process $\mathscr{L}\left(F_{N}\right)_{p_{1} / r_{1}}$ gives an algebra isomorphic to $\mathscr{L}\left(F_{r}\right), r \in Q$, where the integer part of $r$ is $(N-1) k$.

For this reason the next reduction processes will not increase the number of elements of the incomplete semicircular family generating the reduced algebra $\mathscr{L}\left(F_{N}\right)_{p_{j} / q_{j}}$, so that we will obtain by induction incomplete semicircular families, each comprising holes of the (reduced part of the) preceding one.

Easy estimations will give us that the total area of the holes of these incomplete semicircular families tends to zero, and hence by the remarks in the second paragraph, we will obtain at the limit, a free semicircular family.

To show that this family is also a system of generators for the reduced algebra, we will choose, from the beginning, an increasing family of finite sets exhausting the reduced algebra, and we will impose conditions so that the generators of the iterated reduced algebras almost contain these finite sets, always with a better order of approximation.

The induction step that will make possible such a choice of the generators is contained in the following lemma, which is a consequence of Corollary 6.

Lemma 9. Let $\left(X^{0}, X^{1}, \ldots, X^{n}\right)$ be an incomplete semicircular family generating the algebra $\mathscr{A}$ (which is a type $I I_{1}$ factor with trace $\tau$ ). 
Let e be any projection in $\left(X^{0}\right)^{\prime \prime}$, such that $\tau(e) \geqq\left(1-\frac{\alpha}{n}\right)^{1 / 2}$, where $\alpha$ is the total area of the holes of the family. Assume that there exists a projection $e_{0} \in\left(X^{0}\right)^{\prime \prime}$ of rational trace value, $e_{0} \leqq e$ that also covers the holes of the family.

Then for any $\varepsilon>0$, and any finite set $F \subseteq e \mathscr{A}$, there exists $\delta_{\varepsilon}>0$ such that for each projection $e_{1} \in\left(X^{0}\right)^{\prime \prime}$, of rational trace value, $\mathrm{e}_{1} \geqq \mathrm{e}$ and $\tau\left(e_{1}-e\right) \leqq \delta_{\varepsilon}$, we can find an incomplete semicircular family

$$
\left(c_{1}^{-1 / 2} e_{1} X^{0} e_{1}, \ldots, c_{1}^{-1 / 2} e_{1} X^{n-1} e_{1}, c_{1}^{-1 / 2} Y^{n}\right)
$$

in $e_{1} \mathscr{A} e_{1}$ (where $\left.c_{1}=\tau\left(e_{1}\right)\right)$, covering holes of $\left(c_{1}^{-1 / 2} e_{1} X^{i} e_{1}\right)_{i=0}^{n}$ and such that $F$ is $\varepsilon$-almost contained in

$$
\left\{\left(e X^{0} e, \ldots, e X^{n-1} e, e Y^{n} e\right)\right\}^{\prime \prime}
$$

with respect to norm \|\|$_{\tau}$.

Proof. The algebra $e \mathscr{A} e$ is generated by elements of the form $e X^{i_{1}} \ldots X^{i_{m}} e$, $m \geqq 1, i_{1}, \ldots, i_{m} \in\{1,2, \ldots, n\}$. By taking the linear span we may assume that all elements in $F$ are of this form.

A trivial continuity argument shows that for every $\varepsilon>0$, there exists $\delta=\delta_{\varepsilon}$, such that for any projection $g$ in $\left(X^{0}\right)^{\prime \prime}$ with $\tau(1-g)<\delta_{\varepsilon}$ the elements in the set $F_{g}$, obtained by replacing in $F$ a monomial $e X^{i_{1}} \ldots X^{i_{m}} e$ by $e g X^{i_{1}} \ldots g X^{i_{m}} g e$, are at distance (with respect to \|\|$_{\tau}$ ) less than $\varepsilon$ to the corresponding elements in $F$.

We choose projections $e_{ \pm}$of rational trace value in $\left(X^{0}\right)^{\prime \prime}$ such that $e_{+} \geqq e \geqq e_{-} \geqq e_{0}, \tau\left(e_{+}-e_{-}\right) \leqq \delta_{\varepsilon}$ and let $g=\left(1-e_{+}\right)+e_{-}$.

By Corollary 6 of Lemma 5, we obtain that there exists an incomplete semicircular family $\left(c_{+}^{-1 / 2} e_{+} X^{0} e_{+}, \ldots, c_{+}^{-1 / 2} e_{+} X^{n-1} e_{+}, c_{+}^{-1 / 2} Y^{n}\right)$ in $e_{+} \mathscr{A} e_{+}$covering holes of $\left(c_{+}^{-1 / 2} e_{+} X^{i} e_{+}\right)_{i=0}^{n}\left(c_{+}=\tau\left(e_{+}\right)\right)$and generating $e_{+} \mathscr{A} e_{+}$.

Moreover the algebra $B_{g}=\left\{\left(g X^{s} g\right)_{s=0}^{n}\right\}^{\prime \prime}$ has the property that

$$
e_{-} B_{g} e_{-} \subseteq\left\{\left(e_{-} X^{i} e_{-}\right)_{i=0}^{n-1} \cup\left\{e_{-} Y^{n} e_{-}\right\}\right\}^{\prime \prime} .
$$

On the other hand, we obviously have that $F_{g} \subseteq e_{-} B_{g} e_{-}$, and hence we get

$$
F_{g} \subseteq\left\{\left(e_{-} X^{i} e_{-}\right)_{i=0}^{n-1} \cup\left\{e_{-} Y^{n} e_{-}\right\}\right\}^{\prime \prime} \subseteq\left\{\left(e X^{i} e\right)_{i=0}^{n-1} \cup\left\{e Y^{n} e\right\}\right\}^{\prime \prime} ;
$$

the last inclusion is consequent to the fact that $e_{-} \leqq e$, and $e_{-}, e \in\left(X^{0}\right)^{\prime \prime}$. Since the elements in $F$ were at distance less than $\varepsilon$, with respect to the corresponding elements in $F_{g}$, the conclusion follows, by choosing $e_{1}=e_{+}$. Q.E.D.

We can now prove our main theorem.

Theorem 10. $\mathscr{L}\left(F_{N}\right)_{\frac{1}{\sqrt{k}}} \cong \mathscr{L}\left(F_{(N-1) k+1}\right), k, N \in \mathbb{N}, N \geqq 2$.

Proof. Let $\left(Z^{1}, Z^{2}, \ldots, Z^{N}\right)$ be a free semicircular family generating $\mathscr{A}=\mathscr{L}\left(F_{N}\right)$. Take a rational approximation $\frac{r_{1}}{p_{1}}$ of $\sqrt{k}$, such that

$$
\frac{\sqrt{k}}{2} \leqq \frac{r_{1}}{p_{1}} \leqq \sqrt{k} ; \quad\left|\frac{r_{1}}{p_{1}}-\sqrt{k}\right| \leqq \frac{1}{p_{1}} \text {. }
$$


We will have that

$$
\begin{aligned}
(N-1)\left(k-\frac{r_{1}^{2}}{p_{1}^{2}}\right) & =(N-1)\left(\sqrt{k}-\frac{r_{1}}{p_{1}}\right)\left(\sqrt{k}+\frac{r_{1}}{p_{1}}\right) \\
& \leqq 2 \sqrt{k}(N-1) \cdot \frac{1}{p_{1}} .
\end{aligned}
$$

Choosing $\frac{r_{1}}{p_{1}}$ so that $p_{1}$ is big enough, it will follow that we may assume that

$$
p_{1}^{2}(N-1)\left(k-\frac{r_{1}^{2}}{p_{1}^{2}}\right) \leqq \frac{p_{1}^{2}}{4}
$$

We will also choose $\frac{r_{1}}{p_{1}}$ sufficiently close to $\sqrt{k}$, so that the integer part $n$ of $(N-1) \frac{r_{1}^{2}}{p_{1}^{2}}+1$ be $(N-1) k$. By Corollary 8 it will follows that, whenever $e_{1} \in\left(Z^{1}\right)^{\prime \prime}$ is a projection of trace $\tau\left(e_{1}\right)=\frac{p_{1}}{r_{1}}$, the algebra $e_{1} \mathscr{A} e_{1}$ is generated by an incomplete semicircular family $\left(X_{1}^{0}, X_{1}^{1}, \ldots, X_{1}^{n}\right) \subseteq e_{1} \mathscr{A} e_{1}$, with total area of the holes $(N-1)\left(k-\tau\left(e_{1}\right)^{-2}\right)$.

Because of the assumption on $p_{1}$, we may choose this incomplete semicircular family so that there exists a projection $e_{0}$ in $\left(X_{1}^{0}\right)^{\prime \prime}=e_{1}\left(Z^{1}\right)^{\prime \prime} e_{1}$ of relative trace (in $\left.e_{1} \mathscr{A} e_{1}\right), \quad \tau_{e_{1}}\left(e_{0}\right)=\frac{\tau\left(e_{0}\right)}{\tau\left(e_{1}\right)}=\frac{1}{2}$, and such that $e_{0}$ covers the holes of $\left(X_{1}^{0}, X_{1}^{1}, \ldots, X_{1}^{n}\right)$. The property of $p_{1}$ used here was that

$$
p_{1}^{2}(N-1)\left(k-\left(p_{1} / r_{1}\right)^{-2}\right) \leqq p_{1}^{2} / 4 .
$$

Such a selection is possible due to the freedom in choosing the places of the holes of an incomplete semicircular family generating the reduced algebra, which in turn is due to the freedom of the choice of the functions $p(i, j, \alpha), q(i, j, \alpha)$ in Lemma 1. One may also use here the procedure described in Lemma 5 , or one may use the uniqueness up to isomorphism of the algebra $e_{1} \mathscr{A} e_{1} \cong \mathscr{L}\left(F_{M_{1}}\right)$, $M_{1}=(N-1)\left(r_{1}^{2} / p_{1}^{2}\right)+1$, to find a family of generators of $e_{1} \mathscr{A} e_{1}$ with the required properties, or one can use directly Corollary 8 . Moreover since $\frac{1}{\sqrt{k}} \tau\left(e_{1}\right)^{-1}=$ $\frac{1}{\sqrt{k}} \frac{r_{1}}{p_{1}}>\frac{1}{2}=\tau_{e_{1}}\left(e_{0}\right)$ it follows that we are also able to choose a projection $e$ in $\left(X^{0}\right)^{\prime \prime}$ of absolute trace $\tau(e)=\frac{1}{\sqrt{k}}, e_{0} \leqq e \leqq e_{1}$.

Having made these choices, we choose a decreasing family of projections $\left(e_{s}\right)_{s \in \mathbb{N}}$ in $\left(X_{1}^{0}\right)^{\prime \prime}, e_{s} \downarrow e$, of rational trace $\tau\left(e_{s}\right)=\frac{p_{s}}{r_{s}}, s \in \mathbb{N}$, where $\delta_{s}=\tau\left(e_{s}-e\right)$ will be chosen small enough.

We also start with an increasing family of finite sets $F_{1} \subseteq F_{2} \subseteq \cdots F_{s} \subseteq e \mathscr{A} e$, and a sequence $\left(\varepsilon_{s}\right)_{s \in \mathbb{N}}$ of positive real numbers, $\varepsilon_{s} \downarrow 0$.

We will show by induction that it is possible to find the projection $e_{s}$, and an incomplete semicircular family $\left(c_{s}^{-1 / 2} X_{s}^{0}, \ldots, c_{s}^{-1 / 2} X_{s}^{n}\right)$ in $e_{s} \mathscr{A} e_{s}$, covering holes 
of the preceding family.

$$
\left(c_{s}^{-1 / 2} e_{s} X_{s-1}^{i} e_{s}\right)_{i=0}^{n}
$$

with total area of the holes

$$
\alpha_{s}=(N-1)\left(k-r_{s}^{2} / p_{s}^{2}\right)
$$

and such that $F_{s}$ is $\varepsilon_{s}$-almost contained in the algebra $\left\{\left(e X_{s}^{i} e\right)_{i=0}^{n}\right\}^{\prime \prime}$. Here $c_{s}=\tau\left(e_{s}\right)$, while $X_{s}^{i}=e_{s} X_{s-1}^{i} e_{s}$, for $i=0,1, \ldots, n-1$.

Indeed if we found such a family for $s$, we may find the corresponding family for the next index $s+1$, as follows:

Since $\left.\tau_{e_{s}}(e)=\frac{\tau(e)}{\tau\left(e_{s}\right.}\right)=\left(1-\alpha_{s} / n\right)^{1 / 2} \quad$ (as this is equivalent to $t^{2} / k=$ $1-(N-1)\left(k-t^{2}\right) n^{-1}$ which is true since $n=(N-1) k$, if $\left.t=r_{s} / p_{s}=\tau\left(e_{s}\right)^{-1}\right)$ and since $e \geqq e_{0}, e_{0}$ covers the holes of $\left(c_{s}^{-1 / 2} X_{s}^{i}\right)_{i=0}^{n} \subseteq e_{s} \mathscr{A} e_{s}$, it follows by Lemma 9 , at the beginning of this paragraph, that if $e_{s+1} \geqq e$ is close enough to $e\left(e_{s+1} \leqq e_{s}\right)$ (i.e. $\tau\left(e_{s+1}-e\right) \leqq \delta_{s+1}$ is sufficiently small) then there exists a family $\left(X_{s+1}^{i}\right)$ in $e_{s+1} \mathscr{A} e_{s+1}$ with the required properties.

The only thing that one has to check is the formula for the total area of the holes $\alpha_{s+1}$ of this new family.

But by Lemma 5 we have

$$
\alpha_{s+1}=n-\left(n-\alpha_{s}\right)\left[\tau_{e_{s}}\left(e_{s+1}\right)\right]^{-2}=n-\left[n-(N-1)\left(k-t^{2}\right)\right] \frac{a^{2}}{t^{2}},
$$

where $t=\frac{r_{s}}{p_{s}}=\tau\left(e_{s}\right)^{-1} ; a=\frac{r_{s+1}}{p_{s+1}}=\tau\left(e_{s+1}\right)^{-1}$.

Since $n=k(N-1)$ we get that

$$
\alpha_{s+1}=(N-1)\left[k-\left(k-\left(k-t^{2}\right)\right) a^{2} / t^{2}\right]=(N-1)\left[k-a^{2}\right],
$$

which is exactly the formula we were looking for.

Hence the induction step works, and we want to prove that the sequence

$$
\left(e X_{s}^{n} e\right)_{s \in \mathbb{N}}
$$

is Cauchy in the norm $\|\cdot\|_{\tau}$.

But if $a \geqq b, a, b \in \mathbb{N}$ and $\tau_{a}$ is the relative trace on $e_{a} \mathscr{A} e_{a}, \tau_{a}(x)=\frac{\tau(x)}{\tau\left(e_{a}\right)}$, for $x$ in $e_{a} \mathscr{A} e_{a}$, then, since the difference between the elements $e_{a} X_{b}^{n} e_{a}$ and $X_{a}^{n}$ is concentrated within the projection $e$, it follows (using also Remark 1 and the formulas for the total area of the holes $\alpha_{a}, \alpha_{b}$ ) that

$$
\begin{aligned}
\left\|e X_{a}^{n} e-e X_{b}^{n} e\right\|_{\tau_{a}}^{2} & \leqq\left\|X_{a}^{n}-e_{a} X_{b}^{n} e_{a}\right\|_{\tau_{a}}^{2} \\
& =(N-1)\left(k-\left(r_{b} / p_{b}\right)^{2}-k-\left(r_{a} / p_{a}\right)^{2}\right) \\
& =(N-1)\left[\left(r_{a} / p_{a}\right)^{2}-\left(r_{b} / p_{b}\right)^{2}\right]
\end{aligned}
$$

Since all the norms \|\|$_{\tau_{a}}, a \in \mathbb{N}$ are uniformly equivalent (as $\left(\tau\left(e_{a}\right) \rightarrow \tau(e) \neq 0\right.$ ) and since $\left(r_{a} / p_{a}\right)_{a \in \mathbb{N}}$ converges to $\sqrt{k}$, it follows that the sequence $\left(e X_{a}^{n} e\right)_{a \in \mathbb{N}}$ is Cauchy in the norm \|\|$_{\tau}$. 
We have $\left\{\left(e X_{a}^{i} e\right)_{i=0}^{n}\right\}^{\prime \prime} \subseteq\left\{\left(e X_{a+1}^{i}\right)_{i=0}^{n}\right\}^{\prime \prime}$ since $\left(\tau\left(e_{a+1}\right)^{-1 / 2} X_{a+1}^{i}\right)_{i=0}^{n}$ covers holes of $\left(\tau\left(e_{a+1}\right)^{-1 / 2} e_{a+1} X_{a}^{i} e_{a+1}\right)_{i=0}^{n}$. In fact

$$
e_{a+1} X_{a}^{n} e_{a+1}=X_{a+1}^{n}-\sum_{r=1}^{l} f_{i_{r}} X_{a+1}^{n} f_{j_{r}},
$$

is the formula for recovering the holes of $e_{a+1} X_{a}^{n} e_{a+1}$ from $X_{a+1}^{n}$, where $f_{i_{r}}, f_{j_{r}}$ for $r=1, \ldots, l$ are projections in $e_{0}\left(X_{a}^{0}\right)^{\prime \prime} e_{0}$. The construction of the sequence

$$
\left(X_{b}^{n}\right)_{b \in \mathbb{N}, b \geqq a},
$$

shows that this formula (under $e$ ) remains valid for any $b$, i.e. for $b \geqq a+1$, we have

$$
e X_{a}^{n} e=e X_{b}^{n} e-\sum_{r=1}^{l} f_{i_{r}} X_{b}^{n} f_{j_{r}},
$$

so that when $b$ tends to infinity we get

$$
e X_{a}^{n} e=X^{n}-\sum_{r=1}^{t} f_{i_{r}} X^{n} f_{j_{r}}
$$

where $f_{i_{r}}, f_{j_{r}}$ are projections in $e_{0}\left(X_{a}^{0}\right)^{\prime \prime} e_{0} \subseteq\left(X^{0}\right)^{\prime \prime}$, where $X^{n}$ is the limit, after $a$, of $e X_{a}^{n} e$.

Therefore $\bigcup_{s} F_{s}$, is contained in $\left\{\left(e X^{i} e\right)_{i=0}^{n}\right\}^{\prime \prime}$, where $X^{i}=e X_{a+1}^{i} e=e X_{a}^{i} e$, for any $a \in \mathbb{N}, i=0, \ldots, n-1$.

Hence $e \mathscr{A} e=\left\{\left(e X^{i} e\right)_{i=0}^{n}\right\}^{\prime \prime}$. Finally, the family $\left(\tau(e)^{-1 / 2} X^{i}\right)_{i=0}^{n}$ is free and semicircular, as the total area of the holes of the family $\left(\tau\left(e_{a}\right)^{-1 / 2} e_{a} X_{a}^{i} e_{a}\right)_{i=0}^{n}$ in $e_{a} \mathscr{A} e_{a}$ tends to zero, so that by Remark 2, the limit family $\left(\tau(e)^{-1 / 2} X^{i}\right)_{i=0}^{n}$ is semicircular. This ends the proof of the theorem. Q.E.D.

As we mentioned in the introduction, the well known elementary technique in type II factors, $[1,5]$ give (out of this theorem) the following corollaries.

Corolary 11. $\mathscr{L}\left(F_{2}\right) \otimes B(H)$ is isomorphic to $\mathscr{L}\left(F_{k+1}\right) \otimes B(H)$ for each $k \in \mathbb{N}$.

Since the isomorphism class of the algebra $\mathscr{L}\left(F_{N}\right) \otimes B(H)$ for finite positive integer (or even rational) $N$ is thus independent of $N$, it then follows that the fundamental group $\mathscr{F}\left(\mathscr{L}\left(F_{N}\right)\right)$ cannot distinguish between the algebras $\mathscr{L}\left(F_{N}\right)$, for finite $N$.

Corollary 12. The multiplicative subgroup of $\left(\mathbb{R}_{+} \backslash\{0\}\right), \mathscr{F}\left(\mathscr{L}\left(F_{N}\right)\right)$ is independent of $N,(N \in \mathbb{N}, N$ finite, $N \geqq 2)$.

Proof. This comes from the definition of $([1])$ of the fundamental group of a $\mathscr{F}(M)$ type $\mathrm{II}_{1}$ factor $M$, with normalized trace $\tau$, as the set

$$
\mathscr{F}(M)=\{t>0 \mid(\exists) \theta \in \operatorname{Aut}(M \otimes B(H)), \theta \text { scales the trace by } t\} .
$$

Here $H$ is a separable Hilbert space, $\varphi$ is the canonical trace on $B(H), M \otimes B(H)$ is endowed with the canonical tensor product trace $T=\tau \otimes \varphi$, while $\theta$ scales trace by $t$ iff

$$
T(\theta(x))=t T(x), \text { for } x \text { in }(M \otimes B(H))_{+} .
$$

The following corollary to Theorem 10 was pointed out by G. Skandalis. 
Corollary. Let $a, b, c, d$ positive integers with $a, b, c, d \geqq 2$ (where $a$ and $c$ may be infinite) and so that

$$
(a-1)(b-1)=(c-1)(d-1)
$$

Then

$$
\mathscr{L}\left(F_{a}\right) \otimes \mathscr{L}\left(F_{b}\right) \cong \mathscr{L}\left(F_{c}\right) \otimes \mathscr{L}\left(F_{d}\right) .
$$

Proof. This a consequence of the following well known isomorphism

$$
M_{t} \otimes N_{1 / t} \cong M \otimes N, \quad t \in \mathbb{R}, \quad t>0 .
$$

Here $M, N$ are type $I I_{1}$ factors.

Note added in proof. After this paper has been widely circulated as an I.H.E.S Preprint, December 1991, further progress has been made to the subject of this paper.

A series of type $I I_{1}$ factors $\mathscr{L}\left(F_{r}\right)$ for real $r>1$ has been introduced with the properties that $\left(\mathscr{L}\left(F_{r}\right)\right)_{r>1}$ is consistent with our definition for $r \in \mathbb{Q}, r>1,\left(\mathscr{L}\left(F_{r}\right)\right)_{t} \cong \mathscr{L}\left(F_{(r-1) t^{-2}+1}\right)$ and $\mathscr{L}\left(F_{r}\right) * \mathscr{L}\left(F_{p}\right) \cong \mathscr{L}\left(F_{r+p}\right), t, r, p \in \mathbb{R}, r, p>1, t>0$. This was independently discovered by $\mathrm{K}$. Dykema (in [8]) and the author (in [10]).

\section{References}

1. Connes, A.: A survey of foliations and operator algebras. Proc. Symp. Pure Math. 38, Part I, 521-628 (1982)

2. Kadison, R.V.: List of open problems at the Baton Rouge Conference. Mimeographed notes 1973

3. Murray, F.J., von Neumann, J.: On rings of Operators. Ann. Math. 44, 716-808 (1943)

4. Rădulescu, F.: The fundamental group of $\mathscr{L}\left(F_{\infty}\right)$ is $\mathbb{R}_{+} \backslash\{0\}$. J. A.M.S., 5, nr. 3, 517-532 (1992)

5. Takesaki, M.: Theory of Operator Algebras, I, II. New York: Springer 1979

6. Voiculescu, D.: Limit laws for random matrices and free products. Invent. Maths. 104, 201-220 (1991)

7. Voiculescu, D.: Circular and semicircular systems and free product factors. In: Operator Algebras, Unitary Representations, Enveloping Algebras. Progress in Math. Vol. 92, Basel, Boston: Birkhäuser, pp. 45-60, 1990

8. Dykema, K.: Interpolated group factors. Preprint, U.C. Berkeley, March 1992. To appear in Pacific Journal of Mathematics

9. Sakai, S.: $C^{*}$-Algebras and $W^{*}$-Algebras. Berlin, Heidelberg, New York; Springer 1971

10. Florin Rădulescu: Random Matrices, Amalgamated free products and subfactors of the von Neumann algebra of a free group. Preprint I.H.E.S. Nr. 89, December 1991

Communicated by H. Araki 